\title{
Antibiotic PK/PD research in critically ill neonates and children: how do we proceed?
}

\author{
Anne Smits*,1 \& Pieter A J G De Cock ${ }^{2,3,4}$ \\ ${ }^{1}$ Neonatal Intensive Care Unit, University Hospitals Leuven, Herestraat 49, 3000 Leuven, Belgium \\ ${ }^{2}$ Department of Pharmacy, Ghent University Hospital, Ghent, Belgium \\ ${ }^{3}$ Heymans Institute of Pharmacology, Ghent University, Ghent, Belgium \\ ${ }^{4}$ Department of Paediatric Intensive Care, Ghent University Hospital, Ghent, Belgium \\ * Author for correspondence: Tel.: +32 1634 3565; anne.smits@uzleuven.be
}

First draft submitted: 29 October 2017; Accepted for publication: 1 December 2017; Published online: 26 January 2018

Keywords: antibiotics $\bullet$ critical illness $\bullet$ pharmacokinetics

Antibiotics are one of the most frequently used drugs in critically ill neonates and children. Nevertheless, they are often used off-label in these patients, with the highest incidence in the smallest patients. Laine et al. recently reported that an increase in birth weight significantly decreases the probability of off-label antibiotic use in a tertiary neonatal intensive care unit [1]. In addition, antibiotic prescribing practices are highly variable between intensive care units, due to the lack of evidence-based data on optimal antimicrobial therapy in neonates and children $[2,3]$.

Efficacy of antimicrobial treatment depends on the achievement of therapeutic concentrations at the infection site. In critically ill neonates and children, this achievement of optimal exposure is challenging due to an altered drug disposition. From birth to adulthood, the body composition changes dramatically, mainly reflecting a decrease in body water content and an increase in body fat content. Furthermore, renal and hepatic maturation need to be taken into account. Nephrogenesis is completed by the end of the 34th week of gestation. Postnatally, changes in (intra)renal blood flow contribute to the increase in glomerular filtration rate and clearance of hydrophilic drugs. Concerning hepatic maturation, Phase I and II drug metabolizing enzymes display isoenzyme-specific developmental changes. Overall, all these physiological alterations are most prominent in the first year of life and result in a large between and within patient variability in antimicrobial pharmacokinetics (PK, concentration vs time) [4]. Second, besides growth and maturation, also disease (e.g., sepsis), co-medication and treatment approaches (e.g., whole body cooling, extra-corporeal membrane oxygenation) may impact the PK of antibiotics and target attainment [3].

After illustrating some population-specific (e.g., protein-binding capacity) and disease-specific aspects (e.g., augmented renal clearance) influencing antimicrobial PK in neonatal and/or pediatric intensive care unit patients, we provide future research directions to improve antimicrobial therapy in this specific population.

\section{From population- to disease-related covariates influencing antimicrobial disposition: some examples}

Drug protein binding is of specific interest when evaluating antimicrobial exposure, since only the unbound drug can exert a therapeutic effect [5]. In neonates, the median unbound fraction of cefazolin and flucloxacillin in plasma are reported to be higher compared with adult values [6-8]. Recently, the unbound fraction of vancomycin in critically ill children was also found to be higher compared with adults without criticall illness [9]. The decreased drug-plasma protein binding in neonates and young infants can in part be explained by overall lower albumin concentrations compared with adults; for compounds like cefazolin also an altered binding affinity is considered [10]. In critical illness, protein binding might be additionally impaired due to disease-related hypoalbuminemia. For all the above reasons, integration of protein binding in PK modeling analyses should be promoted.

Furthermore, fluid changes (e.g., hypovolemia, fluid boluses, edema), inflammation, the use of inotropes and augmented renal clearance (ARC) are reported to cause antimicrobial pharmacokinetic alterations and changes in target attainment in critically ill adults [5]. Augmented renal clearance, defined as enhanced renal elimination of a circulating solute, is being described with increasing regularity in critically ill adults and may result in 
subtherapeutic antibiotic exposure. The underlying pathophysiological mechanism of ARC is not yet completely unveiled. However, the biological response to inflammation and infection, combined with the administration of fluid boluses and vasoactive compounds increase cardiac output and renal blood flow, resulting in an increased glomerular filtration rate, are considered to be involved [11]. In critically ill children, ARC was recently described in 50 patients receiving amoxicillin-clavulanic acid [12]. Besides maturational changes, also plasma cystatin C (as renal biomarker) and concomitant treatment with vasopressors (as measure of disease severity, irrespective of renal function) were identified as covariates of amoxicillin clearance. A dose of $25 \mathrm{mg} / \mathrm{kg}$ every $4 \mathrm{~h}$ was needed to achieve therapeutic targets for amoxicillin and clavulanic acid, which is a higher total daily dose compared with available references since ARC was not yet taken into account [12]. To the best of our knowledge, in neonates, reports on ARC are currently not available.

\section{Future perspectives \& remaining challenges}

The introduction of PK modeling and simulation methods, alternative and low-volume PK sampling approaches improved the feasibility of clinical pharmacology research in vulnerable populations. Population PK analyses using nonlinear mixed effects modeling has become the standard method to explore drug disposition in neonates and young children. Recently, 'model-based bridging approaches' have been reported in which PK in younger patients are extrapolated from PK models using data from older children, adults, animals and/or in vitro data. Zhao et al. used this 'bridging approach' to predict neonatal fluconazole PK and dosing [13]. This proof-of-concept study illustrates that combining data can be of added value in predicting antimicrobial exposure in neonates and young children, while limiting the patient burden.

As alternative PK sampling approaches, dried blood spot and scavenged sampling become more popular. Scavenged sampling, defined as the use of remnant blood from laboratory tests of standard care that would otherwise be discarded, has been applied in neonates for population PK analyses of metronidazole, piperacillin and fluconazole [14]. Furthermore, alternative matrices like saliva may be considered to determine antibiotic concentrations. Although, this approach has been studied for therapeutic drug monitoring of specific antibiotics in (healthy) adults, and to a limited extent in children, data are often insufficient to evaluate the suitability. Cross-validation between the standard (plasma) and alternative matrix are needed [15].

Although progression has been made in the field of developmental PK, there are remaining challenges. First, while dosing regimens usually account for changes in growth and maturation, disease-related covariates related to critical illness are often not accounted for and warrant further study. In particular, knowledge on tissue penetration in critically ill neonates and children is required. Therefore, antibiotic concentration measurement at the effect site is of interest (e.g., cerebrospinal fluid by lumbar puncture, bronchial epithelial lining fluid by bronchoalveolar lavage). To measure interstitial tissue concentrations, microdialysis becomes more and more popular. Using this technique, a microdialysis catheter is inserted in the interstitial space of the tissue of interest, after which a physiological solution is continuously infused through the catheter at a fixed rate, which is then collected outside the body. Through a semipermeable membrane at the top of the probe, drugs can diffuse from the interstitial to the perfused fluid, in which the unbound drug concentration can be quantified at specific time points [16]. Although technically challenging and invasive, the method has been used to quantify cefazolin in skeletal muscle in children $[17,18]$. Currently, it is not adapted to be applied in younger children.

Second, dosing regimens derived from population PK modeling and simulation analyses need prospective validation. Wilbaux et al. recently systematically reviewed available neonatal PK models for antibiotics. Although for vancomycin 17 models were published, a prospective validation of these models was absent [19]. This, in part, explains why there is still no consensus on optimal vancomycin dosing recommendations.

Third, although PK models for many antibiotics become currently available, the exposure-response (pharmacodynamics $[\mathrm{PD}]$ ) relationship of these compounds is less well understood in neonates and young children. Harmonization of currently used antimicrobial PD target indices and target values is needed to properly evaluate target attainment of current and alternative dosing regimens. In addition, building more sophisticated mechanistic PK/PD models should be the next step in the antibiotic dose optimization process. In these models, the PK is linked to the microbiological response over time from in vitro or animal infection experiments [20]. Reports on models describing clinical PK/PD relationships in critically ill neonates and young infants are currently limited [19]. Ultimately, the impact of new dosing regimens on morbidity (i.e., short and long-term effects) and mortality should be investigated. 


\section{Financial \& competing interests disclosure}

The authors have no relevant affiliations or financial involvement with any organization or entity with a financial interest in or financial conflict with the subject matter or materials discussed in the manuscript. This includes employment, consultancies, honoraria, stock ownership or options, expert testimony, grants or patents received or pending, or royalties.

No writing assistance was utilized in the production of this manuscript.

\section{References}

1 Laine N, Kaukonen AM, Hoppu K, Airaksinen M, Saxen H. Off-label use of antimicrobials in neonates in a tertiary children's hospital. Eur. J. Clin. Pharmacol. 73, 609-614 (2017).

2 Metsvaht $\mathrm{T}$, Nellis G, Varendi $\mathrm{H}$ et al. High variability in the dosing of commonly used antibiotics revealed by a Europe-wide point prevalence study: implications for research and dissemination. BMC Pediatr. 15, 41 (2015).

3 Kontou A, Sarafidis K, Roilides E. Antimicrobial dosing in neonates. Exp. Rev. Clin. Pharmacol. 10(3), 239-242 (2017).

4 Smits A, Annaert P, Allegaert K. Drug disposition and clinical practice in neonates: cross talk between developmental physiology and pharmacology. Int. J. Pharmaceutics 452, 8-13 (2013).

5 Uldemolins M, Roberts JA, Rello J, Paterson DL, Lipman J. The effects of hypoalbuminaemia on optimizing antibacterial dosing in critically ill patients. Clin. Pharmacokinet. 50(2), 99-110 (2011).

6 Smits A, Kulo A, Verbesselt R et al. Cefazolin plasma protein binding and its covariates in neonates. Eur. J. Clin. Microbiol. Infect. Dis. 31(12), 3359-3365 (2012).

7 Smits A, Roberts JA, Vella-Brincat JW, Allegaert K. Cefazolin plasma protein binding in different human populations: more than cefazolin-albumin interaction. Int. J. Antimicrob. Agents 43(2), 199-200 (2012).

8 Pullen J, Stolk LM, Degraeuwe PL, van Tiel FH, Neef C, Zimmermann LJ. Protein binding of flucloxacillin in neonates. Ther. Drug Monit. 29(3), 279-283 (2007).

9 De Cock PA, Desmet S, De Jaeger A et al. Impact of vancomycin protein binding on target attainment in critically ill children: back to the drawing board? J. Antimicrob. Chemother. 72(3), 801-804 (2017).

10 Decroix MO, Zini R, Chaumeil JC, Tillement JP. Cefazolin serum protein binding and its inhibition by bilirubin, fatty acids and other drugs. Biochem. Pharmacol. 37(14), 2807-2814 (1988).

11 Roberts JA, Joynt GM, Choi GYS, Gomersall CD, Lipman J. How to optimize antimicrobial prescriptions in the intensive care unit: principles of individualized dosing using pharmacokinetics and pharmacodynamics. Int. J. Antimicrob. Agents 39, 187-192 (2012).

12 De Cock AJGP, Standing JF, Barker CI et al. Augmented renal clearance implies a need for increased amoxicillin-clavulanic acid dosing in critically ill children. Antimicrob. Agents Chemother. 59, 7027-7035 (2015).

13 Zhao W, Le Quellec C, Benjamin DK Jr et al. First dose in neonates: are juvenile mice, adults and in vitro-in silico data predictive of neonatal pharmacokinetics of fluconazole. Clin. Pharmacokinet. 53, 1005-1018 (2014).

14 Ku LC, Smith PB. Dosing in neonates: special considerations in physiology and trial design. Pediatr. Res. 77(1), 2-9 (2015).

15 Kiang TK, Ensom MH. A qualitative review on the pharmacokinetics of antibiotics in saliva: implications on clinical pharmacokinetic monitoring in humans. Clin. Pharmacokinet. 55(3), 313-58 (2016).

16 de Lange ECM, de Boer AG, Breimer DD. Methodological issues in microdialysis sampling for pharmacokinetic studies. Adv. Drug Deliv. Rev. 45, 125-148 (2000).

17 Deitchman AN, Heinrichs MT, Khaowroongrueng V, Jadhav SB, Derendorf H. Utility of microdialysis in infectious disease drug development and dose optimization. AAPS 19(2), 334-342 (2017).

18 Hiembauch AS, Nicolson SC, Sisko M et al. Skeletal muscle and plasma concentrations of cefazolin during cardiac surgery in infants. J. Horac. Cardiocasc. Surg. 148, 2634-2641 (2014).

19 Wibaux M, Fuchs A, Samardzic J et al. Pharmacometric approaches to personalize use of primarily renally eliminated antibiotics in preterm and term neonates. J. Clin. Pharmacol. 56(8), 909-935 (2016).

20 Nielsen EI, Friberg LE. Pharmaockinetic-pharmacodynamic modeling of antibacterial drugs. Pharmacol. Rev. 65(3), 1053-1090 (2013). 